\title{
Parkinson Disease Detection Based on Voice and EMG Pattern Classification Method for Indonesian Case Study
}

Farika Putria, Wahyu Caesarendra ${ }^{a \star}$, Elta D Pasmanasarib, Mochammad Ariyanto ${ }^{a}$, Joga D Setiawan ${ }^{\mathrm{a}}$ ${ }^{a}$ Mechanical Engineering Department, Faculty of Engineering, Diponegoro University, Semarang, Indonesia ${ }^{b}$ Neurogical Department, Faculty of Medicine, Diponegoro University, Semarang, Indonesia

* Corresponding author's Email: w.caesarendra@gmail.com

\begin{abstract}
Parkinson disease $(P D)$ detection using pattern recognition method has been presented in literatures. This paper present multi-class $P D$ detection utilizing voice and electromyography (EMG) features of Indonesian subjects. The multiclass classification consists of healthy control, possible stage, probable stage and definite stage. These stages are based on Hughes scale used in Indonesia for $P D$. Voice signals were recorded from 15 people with Parkinson (PWP) and 8 healthy control subjects. Voice and EMG data acquistion were conducted in dr Kariadi General Hospital Semarang, Central Java, Indonesia. Twenty two features are used for voice signal feature extraction and twelve features are emploed for EMG signal. Artificial Neural Network is used as classification method. The results of voice classification show that accuracy for testing step of $94.4 \%$. For EMG classification, the accuracy of testing of $71 \%$.
\end{abstract}

Keywords: Parkinson's disease (PD); Voice signal; Electromyography (EMG) signal; ANN

\section{INTRODUCTION}

Indonesia shows increase in elderly population along years. One of the problem as the growth of elderly population is neurodegenerative disease such as Alzheimer, dementia and Parkinson Disease (PD). PD first describe by Dr James Parkinson in his paper titled "An Essay on The Shaking Palsy" published on 1871 [1]. PD is one of the top ten most common illnesses in Ciptomangunkusumo Hospital (RSCM) Indonesia [2]. PD is clinically detected by motoric symptom such as tremor, bradykinesia, rigidity and postural instability [1].

In Indonesia, PD detection is mainly on clinical examination. This paper aimed to develop an alternative diagnostic method for PD through pattern classification. Previous study presented the classification method for two classes that are healthy and PD [3-5]. Multi-class pattern recognition method for PD stage classification is necessary, since it help neurologist to detect PD symptoms as early as possible. Early detection can help patient for better life quality and productive elderly population.

Hughes scale is used as PD staging in Dr. Kariadi General Hospital Semarang, Indonesia. Hughes scale consists of three stages, possible, probable and definite. Possible stage define as patients show one of main PD symptoms such tremor at rest, rigidity, bradykinesia or postural instability. Probable stage define as patients show two combination of main PD symptoms or the other alternative patients suffer asymmetrical tremor at rest, asymmetrical rigidity or asymmetrical bradykinesia. Definite stage describe as patients suffer three or four combination of main PD symptoms.

Earlier PD detection study utilizing voice features for pattern recognition methods has been presented in $[2,6]$. The 22 voice features used in those papers were obtained from the University of California-Irvine (UCl) data repository. 
Present study employed voice and EMG signal acquired from Indonesian volunteer. The data were obtained by real experiment collaborate with hospital. Some of voice and EMG signals are collected in Dr. Kariadi General Hospital and the EMG signals are collected in the patient's house. The voice and EMG signals were analysed using simple pattern recogntion procesedures: (1) feature extraction and (2) classification. Differ to earliear study, this paper does not include any dimensionality reduction method because Artificial Neural Network (ANN) can classify multiple class with huge number of features input.

\section{Methods}

\subsection{Feature extraction methods for voice signal}

Twenty two voice feature extraction methods are employed in this study The features include: Multi Dimensional Voice Program (MDVP): $\mathrm{Fo}(\mathrm{Hz}), \mathrm{MDVP}$ : Fhi(Hz), MDVP: Flo(Hz), MDVP: jitter(\%), MDVP: jitter(Abs), MDVP: Relative Average Perturbation (RAP), MDVP: Period Perturbation Quotient (PPQ), MDVP: shimmer, MDVP: shimmer (dB), MDVP: APQ, jitter: DDP, shimmer: amplitude perturbation quotient 3 (APQ3), shimmer: APQ5, shimmer: DDA, Noise to Harmonic Ratio (NHR), Harmonic to Noise Ratio (HNR), Recurrent Period Density Entropy (RPDE), Detrended Fluctuation Analysis (DFA), spread1, spread2, correlation dimension (D2), Pitch Period Entropy (PPE). The detail of these features were presented in $[2,6,7]$.

\subsection{Feature extraction methods for EMG signal}

Twelve features for EMG signals were employed in this study. The features include: Integrated EMG (IEMG), Mean Absolute Value (MAV), Variance of EMG (VAR), Root Mean Square (RMS), Log Detector (LOG), Waveform Length (WL), Kurtosis, Skewness, Mean Frequency (MNF), Median Frequency (MDF), Total Power (TTP), Mean Power (MNP). Detail of these features can be found in [8-12].

\subsection{ANN classification}

This paper used ANN which a classification inspired by human neuron. ANN consists of three layers i.e. input layer, hidden layer and output layer. Input layer is feature calculaton result, hidden layer consist of feedforward network with a logarithmic-sigmoid transfer function and output layer is a hyperbolic tangent sigmoid transfer function.

Equation (1) express first output layer of single layer ANN

$$
a^{1}=f^{1}\left(I W p+b^{1}\right)
$$

where $a^{1}$ is output vector from input layer, $p$ is an n-length input vector, $I W$ is input weight matrix, $f^{1}$ is transfer function of hidden layer, and $b^{1}$ is the bias vector of hidden layer.

The first output neuron of the output layer as written in (2)

$$
a^{2}=f^{2}\left(L W\left(f^{1}\left(I W p+b^{1}\right)\right)+b^{2}\right)
$$

where $a^{2}$ is output vector from output layer, $L W$ is output layer weight matrix, $P$ is transfer function of the output layer, and $b^{2}$ is the bias vector of the output layer.

In multi-layer of neurons, the ANN structure comprises of more than one layer. Each layer has weight matrix $W$, input vector $p$, a bias vector $b$, and an output vector $a$. In each layer has function activation or transfer function of layer $f$. Layer on the multi-layer network has different process. A layer which is next to the input is called hidden layer and a layer that is next to the output of network is called output layer. The structure of multi-layer of neurons is presented in Figure.1.

The output of neuron (a) in the first layer can be defined as expressed in (3) 


$$
a^{1}=f\left(I W^{1,1} p^{1}+b^{1}\right)
$$

The output of neuron (a) in the second layer is defined as in (4)

$$
a^{2}=f^{2}\left(L W^{2,1} f^{1}\left(I W^{1,1} p^{1}+b^{1}\right)+b^{2}\right)
$$

Finally, the output of neuron (a) in the third layer can be described as in (5)

$$
a^{2}=f^{3}\left(L W^{3,1} f^{2}\left(L W^{2,1} f^{1}\left(I W^{1,1} p^{1}+b^{1}\right)+b^{2}\right)+b^{3}\right)
$$

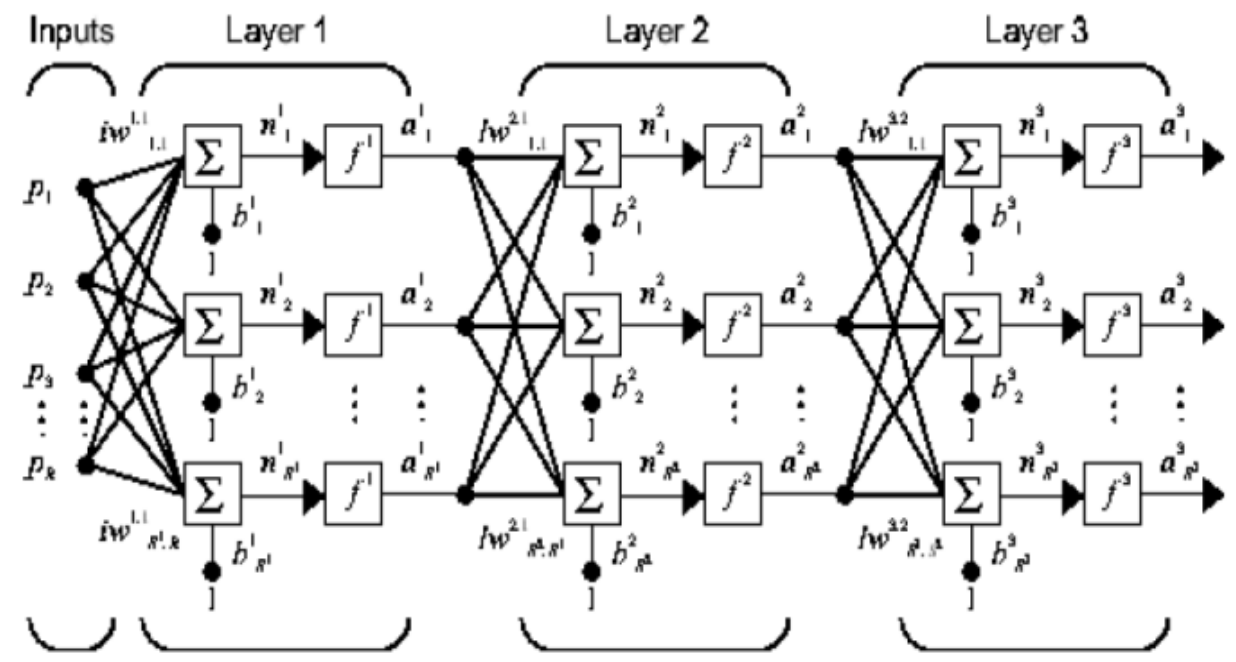

Figure.1 Multi-layer of neurons [13]

The ANN for classification using Mean Square Error (MSE). The MSE measures the magnitude of the forecast errors as shown in (3). Better model will show the smaller values of MSE.

$m s e_{\text {error }}=\frac{\sum\left(y_{1}-y_{2}\right)^{2}}{m}$

\section{Experimental Procedures}

\subsection{Subject participants}

Prior to experiment, a consent form has been issued from dr. Kariadi General Hospital with No. 283/EC/FK-RSDK/2015. Twenty three subjects were participated in this study, 15 people with PD and 8 people as healthy subjects. Table 1 and Table 2 show participants list for patient with PD and healthy people. The voice and EMG signals were acquired from each subject. 


\begin{tabular}{cccc}
\multicolumn{5}{c}{ Table 1. A detail information of PWP } \\
\hline \multicolumn{3}{c}{ People With Parkinson (PWP) } \\
Subject & Sex & Age & $\begin{array}{c}\text { Hughes } \\
\text { Stage }\end{array}$ \\
\hline S01 & M & 68 & Definite \\
S02 & M & 53 & Possible \\
S03 & M & 59 & Possible \\
S04 & F & 79 & Definite \\
S05 & F & 66 & Definite \\
S06 & F & 39 & Probable \\
S07 & F & 58 & Possible \\
S08 & F & 54 & Probable \\
S09 & M & 66 & Probable \\
S10 & M & 80 & Probable \\
S11 & M & 76 & Probable \\
S12 & M & 70 & Definite \\
S13 & M & 68 & Probable \\
S14 & M & 68 & Definite \\
S15 & M & 72 & Definite \\
\hline & & &
\end{tabular}

Table 2. A detail information of healty participants

\begin{tabular}{ccc}
\hline \multicolumn{3}{c}{ Healthy control } \\
Subject & Sex & Age \\
\hline S 16 & F & 71 \\
S 17 & F & 53 \\
S 18 & F & 75 \\
S 19 & M & 70 \\
S 20 & F & 55 \\
S 21 & F & 70 \\
S 22 & M & 52 \\
S 23 & M & 60 \\
S10 & M & 80 \\
S14 & M & 68 \\
S15 & M & 72 \\
\hline
\end{tabular}

\subsection{Experimental procedure of voice signal}

Vocal impairment is one of the earliest symptoms for PD [7]. Dysphonics symptoms often show reduce of loudness, roughness and breathiness. Based on dysphonic symptoms, this paper conduct sustained phonation test for subjects. Sustained phonation test means subjects are instructed to produce single vowel as constant as possible and for as long as possible [7]. 
(a)

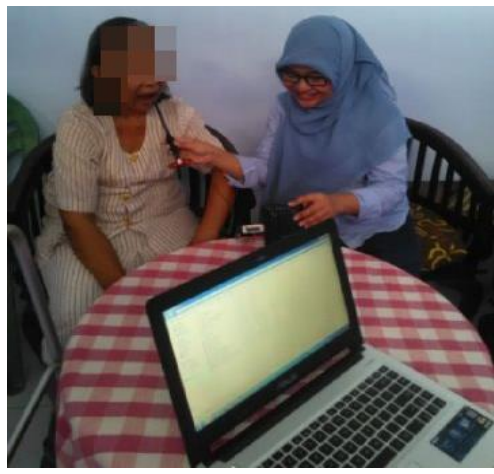

(b)

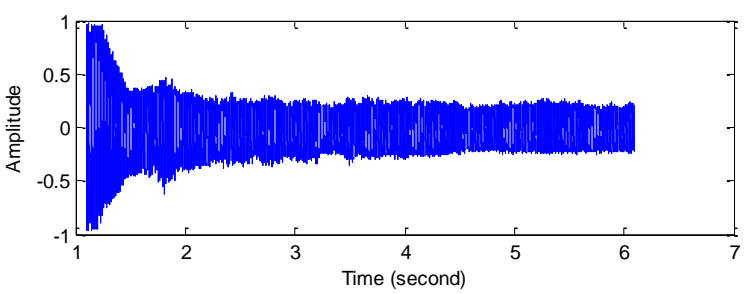

(c)

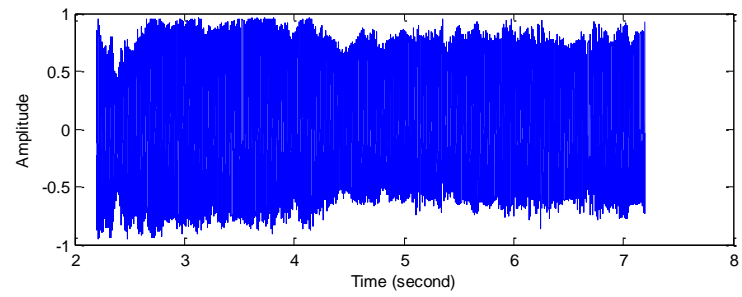

(d)

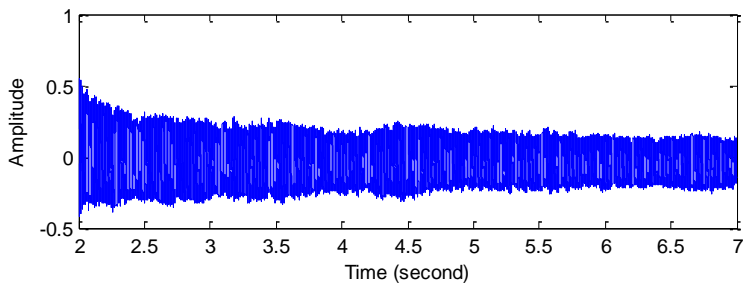

(e)

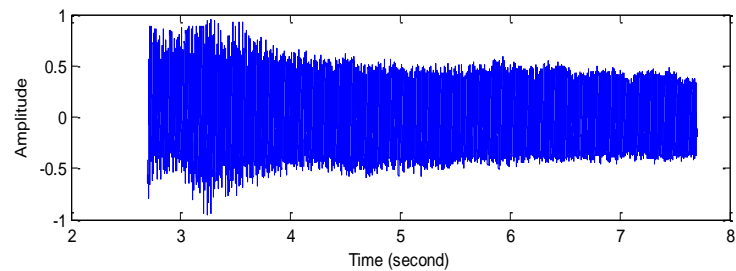

Figure.2 (a) A photograph during voice experiment, (b) Voice signal of healthy subject, (c) Voice signal of PD patient with possible stage, (d) Voice signal of $P D$ patient with probable stage, and (e) Voice signal of $P D$ patient with definite stage.

This research choose "aaa..." vowel to be produced by the subjects during the test. Voice is recorded using uniaxial microphone to noise reduction with $48000 \mathrm{~Hz}$ sampling rate. Fig. 2 an image during experiment and voice signals obtained from participants.

\subsection{Experiment procedure of EMG signal}

The EMG signal was acquired using EMG sensor BITalino plugged kit with sampling rate of $1000 \mathrm{~Hz}$. BITalino plugged kit using bluetooth 2.0 for interface to computer with cover area maximal 10 meter. BITalino can be connected to MATLAB and OpenSignal for EMG signal acquisition.

JEMMME | Journal of Energy, Mechanical, Material, and Manufacturing Engineering 


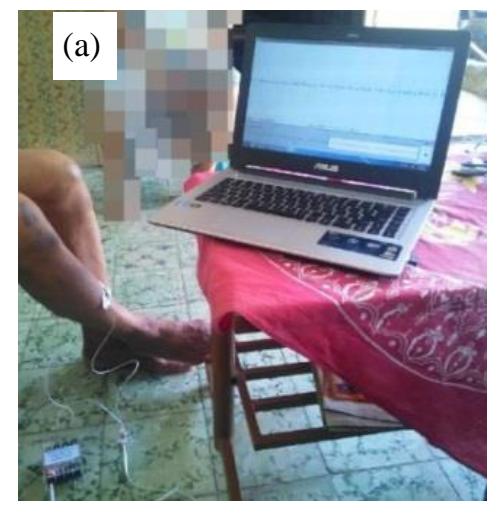

(b)
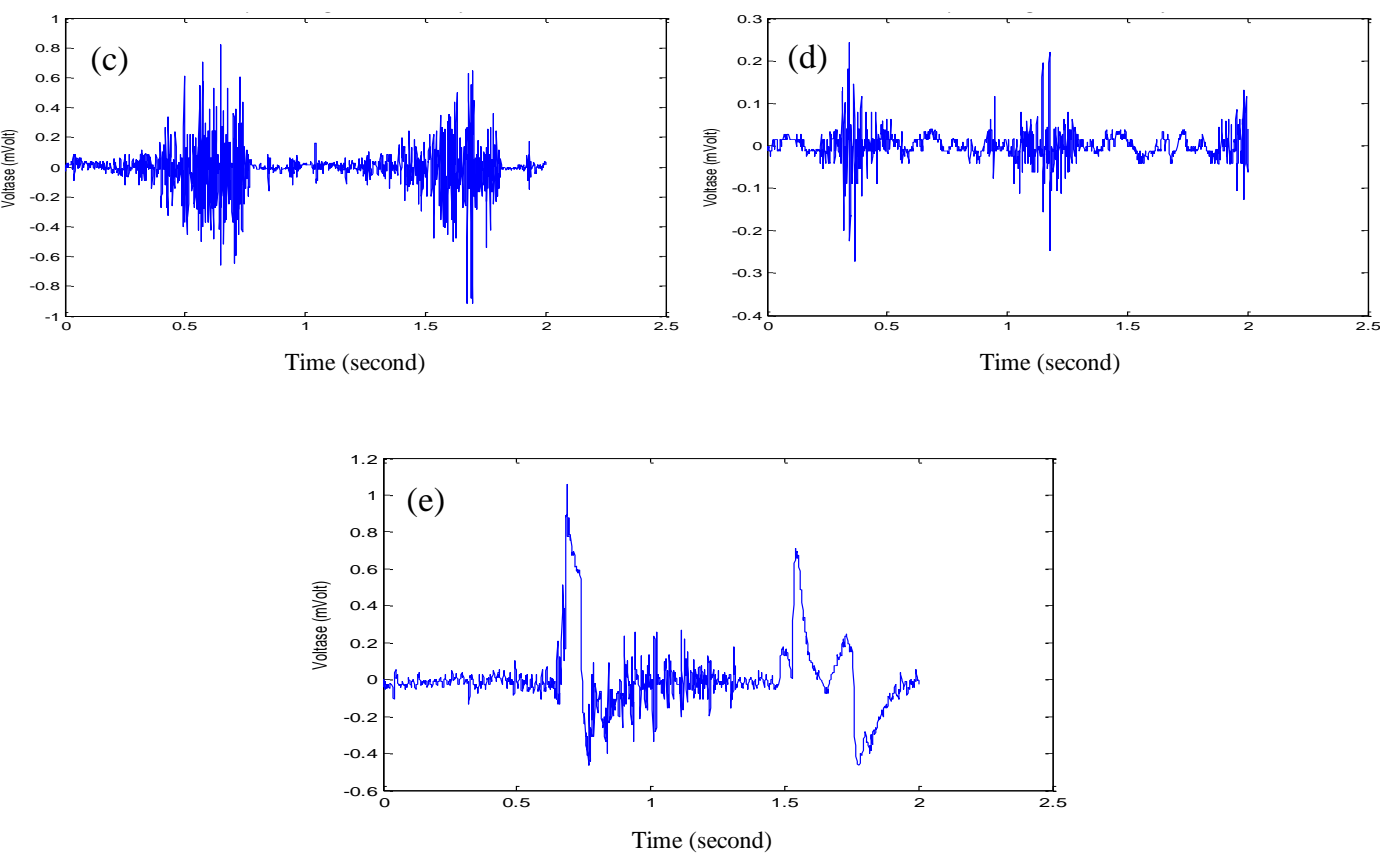

Figure.3 (a) A photograph during EMG experiment, (b) EMG signal of healthy subject (S23), (c) EMG signal of PD patient with possible stage (S02), (d) EMG signal of PD patient with probable stage (S09), and (e) EMG signal of PD patient with definite stage (S14).

The EMG sensor mounted on Tibialis Anterior (centre ground position pad electrode EMG sensor BITalino). Gastrocnemius Medialis (the right position of pad electrode EMG sensor BITalino). The EMG sensor mounted on Tibialis Anterior (centre ground position pad electrode EMG sensor BITalino). Gastrocnemius Medialis (the right position of pad electrode EMG sensor BITalino). The EMG signals were acquired from two EMG experimental works: (1) Tip toe and (2) Twist.

For Tip toe experiment, the subjects were asked to sat and knocked tip of finger and heel on the floor alternately for 20 seconds. Data acqusition was performed for 3 times. For Twist experiment the subjects are asked sat and lift foot about $10 \mathrm{~cm}$ of the floor and twisted ankle about 20 seconds. Data acqusition was performed for 3 times. A photograph of EMG sensor placement during the experiment is presented in Fig. 3(a). The example of EMG signals from healthy subject and three PWP stages are presented in Figs. 3(b)-(e). 


\section{RESULTS AND Discussion}

\subsection{Feature extraction results of voice signals}

An example of two features are presented in Fig. 4. Fig. 4(a) is the mean value of Flo feature collecting from 23 subjects and Fig. 4(b) is the mean value of Jitter Absolute feature collecting from 23 subjects. The blue colour bar represent the PWP and the red colur bar indicate the healthy subjects. It can be seen that there are a different between PWP class and healthy subject.
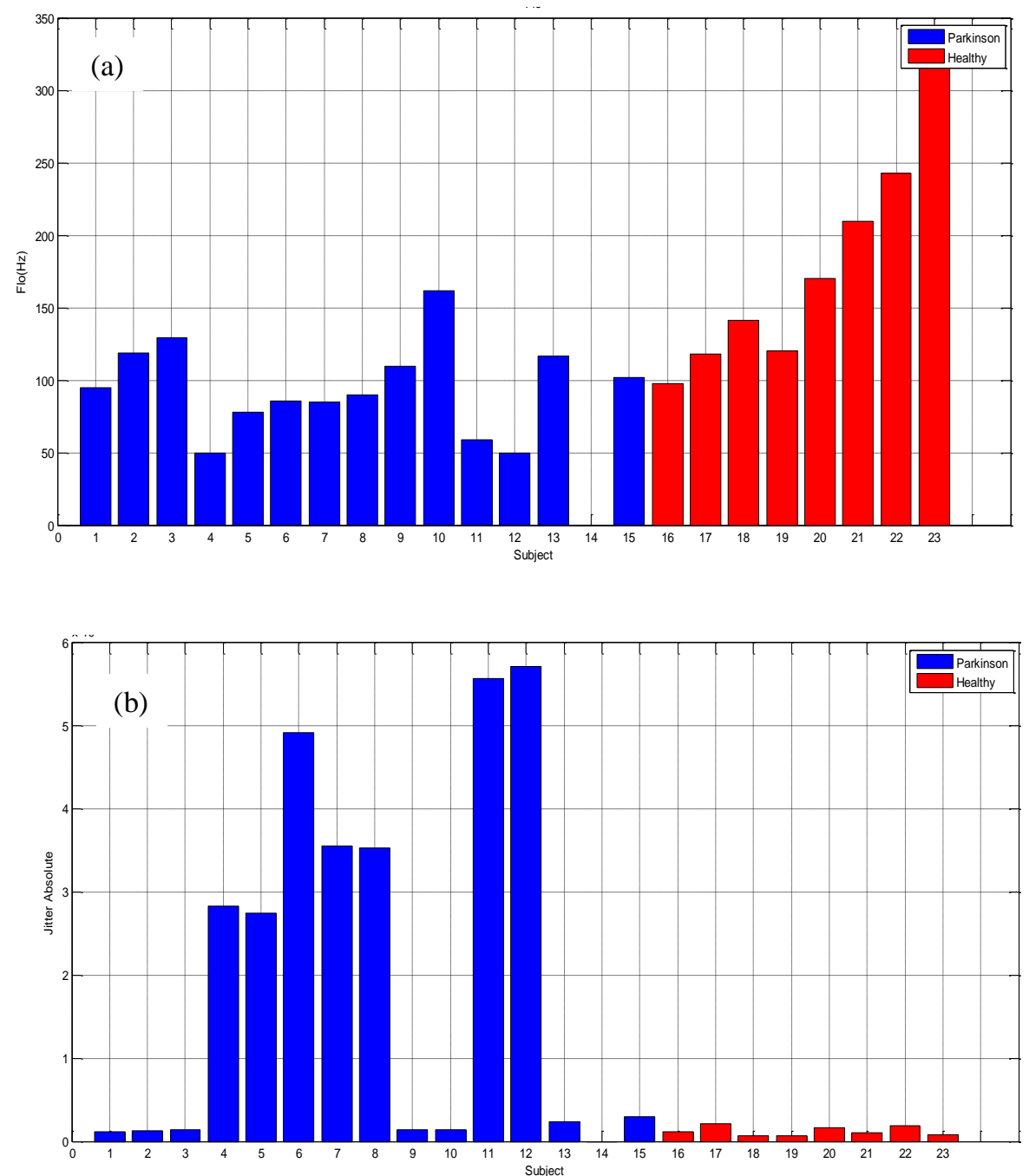

Figure.4 Plot example of two voice features for 23 subjects: (a) Flo feature and (b) Jitter absolute feature

\subsection{Feature extraction results of EMG signals}

Two example of EMG features from tip toe experiment are presented in Fig. 5. Similar to Fig. 4, red bar colour is the healthy subjects and blue bar colour is the PWP subjects. It can be seen that the IEMG feature of healthy subjects are higher than the PWP subjects. In contrast, the kurtosis feature of healthy subjects are lower than PWP subjects.

Two example of EMG features from twist experiment are presented in Fig. 6. Similar to tip toe experiment, the IEMG feature of healthy subjects are higher than the PWP subjects. In contrast, the kurtosis feature of healthy subjects are lower than PWP subjects.

JEMMME | Journal of Energy, Mechanical, Material, and Manufacturing Engineering 

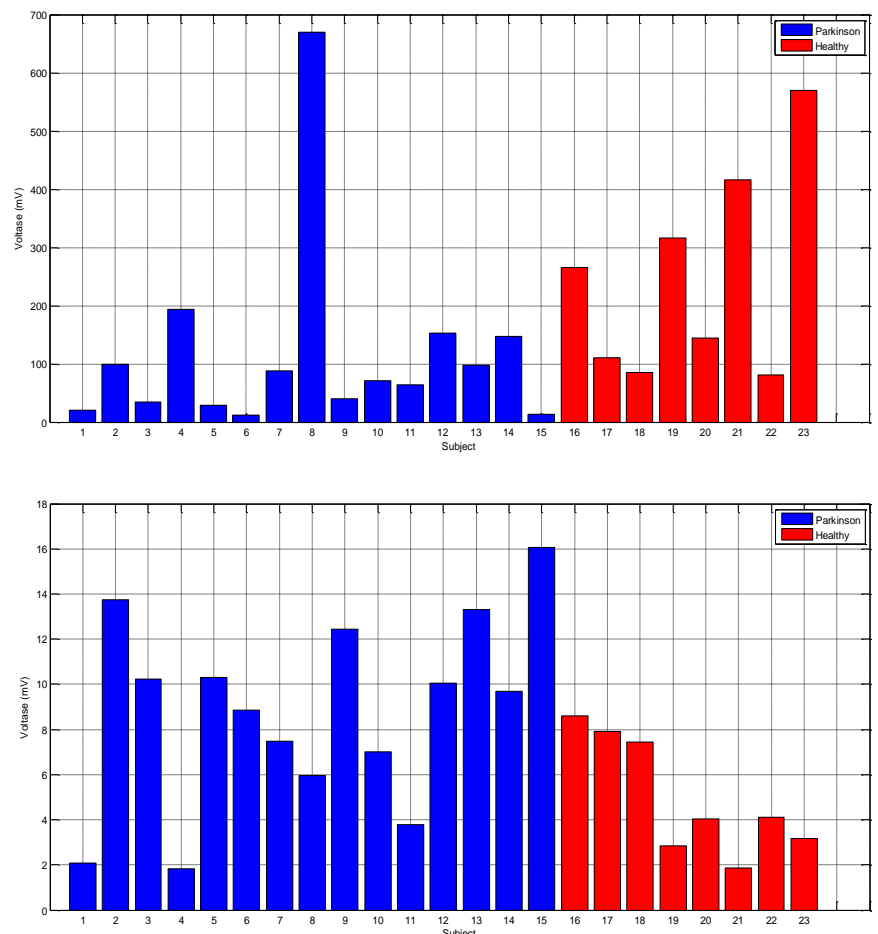

Figure 5. Plot example of EMG feature during tip toe experiment for 23 subjects: (a) IEMG and (b) Kurtosis

According to Fig. 3, the Flo features of healthy subjects (8 subjects) are higher than PWP (15 subjects). In contrast, the Jitter Absoulte features of healthy subjects (8 subjects) are lower than PWP (15 subjects).
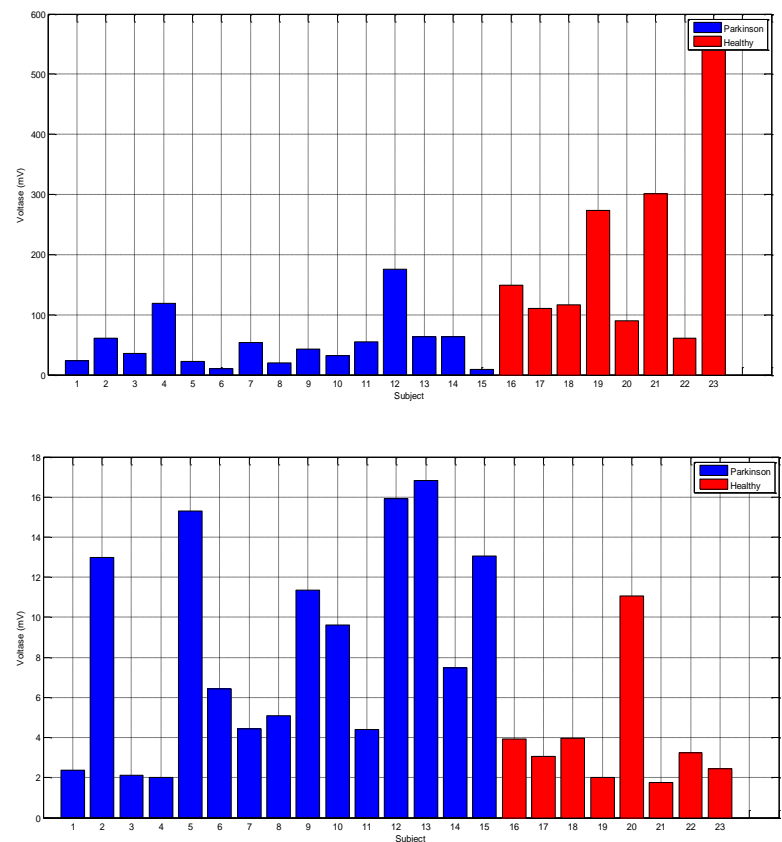

Figure.6 Plot example of EMG feature during twist experiment for 23 subjects: (a) IEMG and (b) Kurtosis

JEMMME | Journal of Energy, Mechanical, Material, and Manufacturing Engineering 


\subsection{Classification results of voice signals}

The classfiying result of ANN method for four classes of voice signals are presented in Fig. 7. For the training result achieved $100 \%$ for target class and output class. Four classes consist of first class for healthy subject, second class for possible Parkinson patient, third class for probable Parkinson patient, and fourth class for definite Parkinson patient.

Fig. 7 shows the classification result of testing step using the ANN method for voice signal for four classes. The accuracy of target class for healthy subject is $57.1 \%$ and for the accuracy of target class is $100 \%$. The accuracy of the target class of second class for possible Parkinson patients is $60 \%$, meanwhile for output class, the accuracy is $100 \%$. The accuracy of the target class of third class for probable Parkinson patients is $100 \%$, meanwhile the accuracy of output class is $25 \%$. The accuracy of the target class of fourth class for different Parkinson patients is $100 \%$, whereas the overall the testing for four.

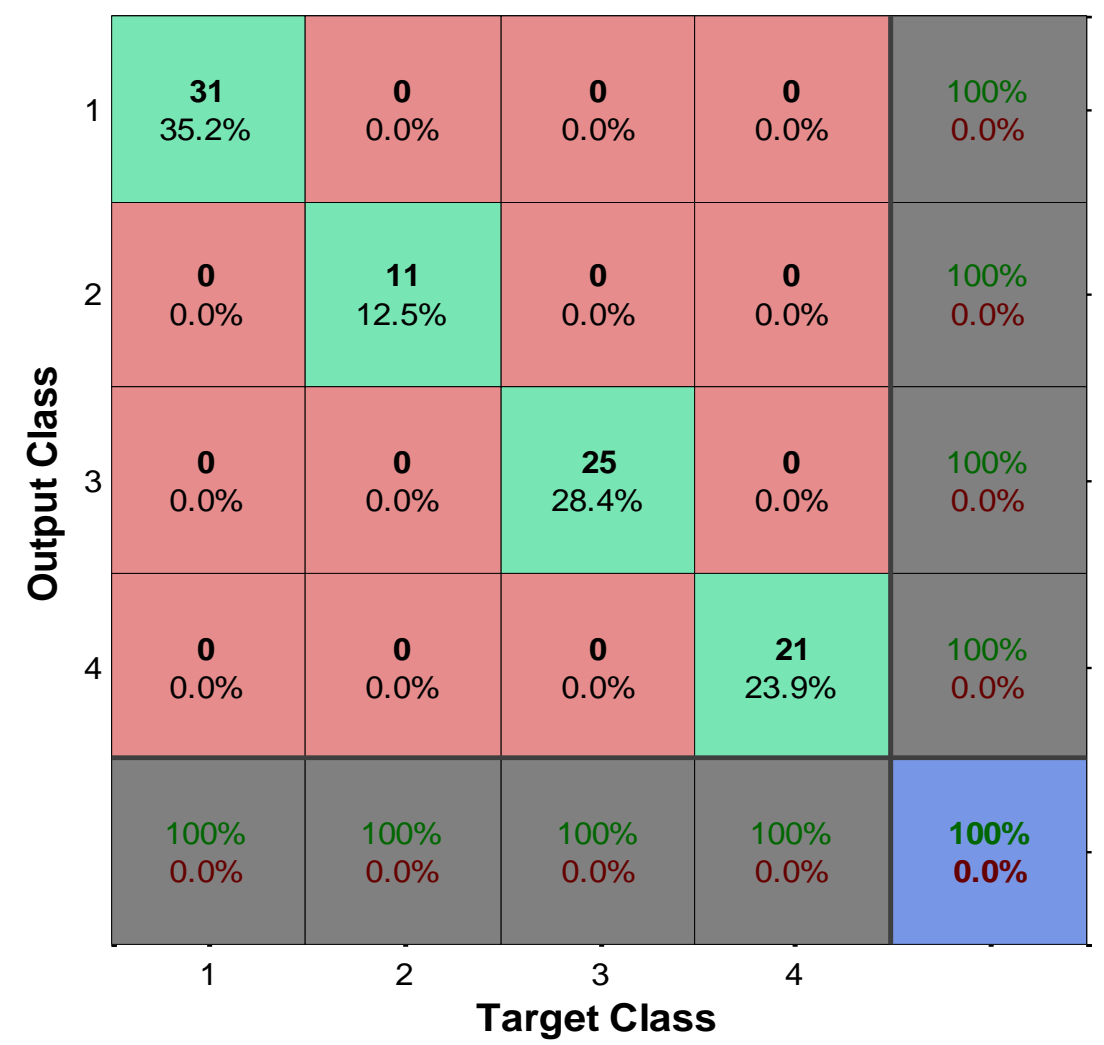

Figure 7. The result of training classification accuracy of voice signal using ANN for 4 classes

Fig. 8 shows the result of total clasification accuracy of four classes voice signal. The accuracy of first class from target class is $91.7 \%$, meanwhile the accuracy of output class is $100 \%$. The accuracy of the second class of target class is $80 \%$, meanwhile output class is $100 \%$. The accuracy of third target class is $100 \%$, meanwhile output class is $90.9 \%$. The accuracy of four target class is $100 \%$, meanwhile for output class $89.2 \%$. Total accuracy of classifying ANN for four classes is $94.4 \%$. Compare to previous classification result [3, 4], the ANN classification has better accuracy result than SVM. However, the comparison is not fair because the previous study used different data for training and testing. 


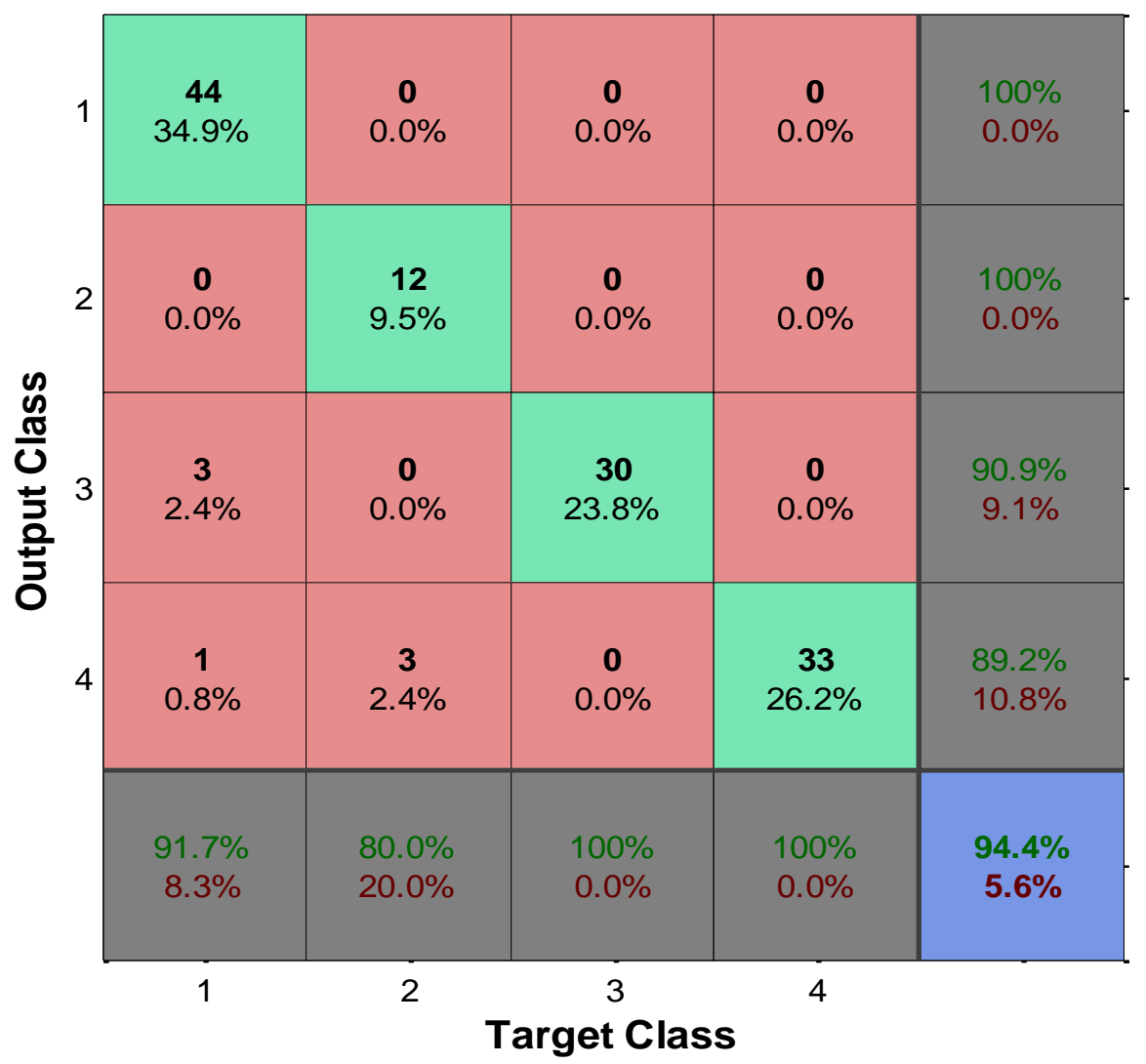

Figure 8. The result of testing classification accuracy of voice signals using ANN for 4 classes

\subsection{Classification results of EMG signals}

The result of ANN classifiying of EMG signal for four classes shown in Fig. 9. The overall accuracy for four classses is $81.6 \%$. the first class for healthy subject, the second class for possible PD subject, the third class for proable PD subject, and fourth class for definite PD subject.

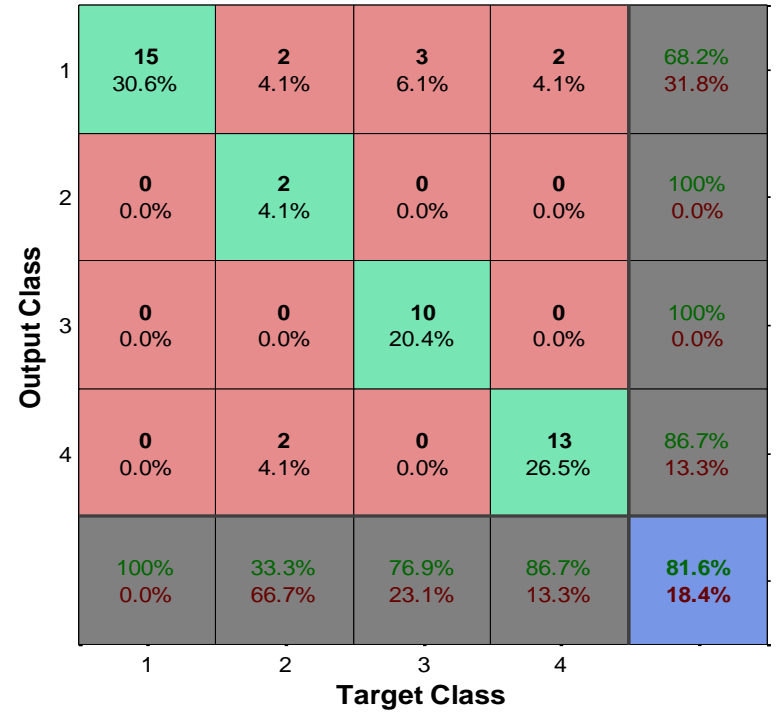

Figure 9. The result of training classifying accuracy of EMG signal using ANN for 4 classes 
The overall accuracy of EMG signal shown in Fig. 10. The overall result of classifying ANN is $71 \%$.

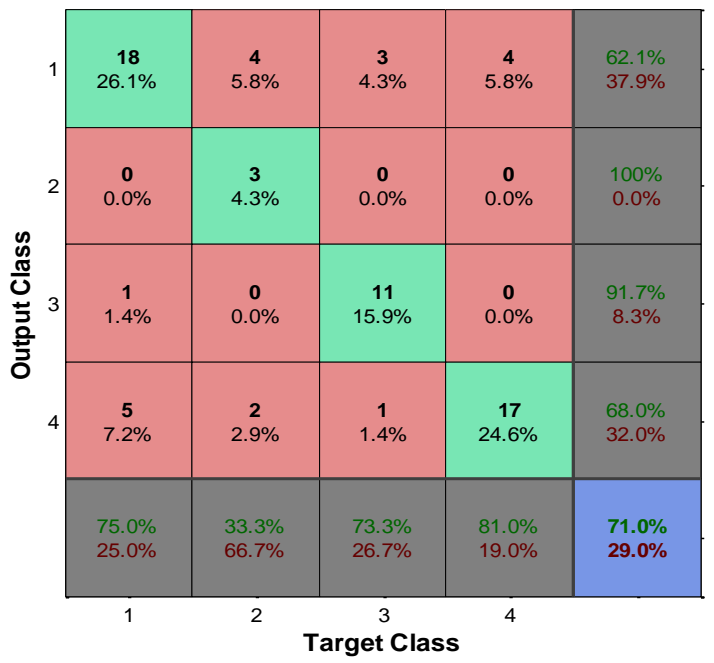

Figure 10. The result of testing classification accuracy of EMG signals using ANN for 4 classes

\section{CONCLUSION}

The pattern classification method for PD detection of Indonesian volunteer based on ANN has been presented. The feature extraction result of voice and EMG signals show that the features between healty subjects and PWP can be distinguished. However, there are some inappropriate features for EMG that can reduce the classification accuracy. The classification result of voice signals for four clasess are better than the classification result of EMG signals. The testing accuracy of voice signals and EMG signals of $94.4 \%$ and $71 \%$, respectively.

\section{Acknowledgements}

This research is funded by INSINAS research fund from Ministry of Research and Higher Education Indonesia 2015. Authors also thank to all participants who willing to contribute for this research. The Authors would also thank to Professor dr. Amin Husni, PAK $(K)$, Sp.S(K), Msc from Neurology Department, Faculty of Medicine, Diponegoro University for kind support during the data acquisition in dr. Kariadi General Hospital.

\section{REFERENCES}

[1] L.I. Golbe, M.H. Mark and J.I. Sage, "Parkinson's Disease Handbook," America: The American Parkinson Disease Association. Inc, 2010.

[2] W. Caesarendra, M. Ariyanto, Joga D Setiawan, Moh. Arozi and Cindy R. Chang, "A Pattern Recognition Method for Stage Classification of Parkinson's Disease Utilizing Voice Features," Second International Conference on Biomedical Engineering (IECBES), 2014.

[3] M. Shahbakhti and D. Taherifar, "Linear and non linear speech features for detection of Parkinson's disease," Biomedical Engineering International Conference, 978-14799-1466-1, 2013.

[4] R.G. Ramani and G. Sivagami, "Parkinson Disease classification using data mining algorithm," International Journal of Computer Applications, vol. 32, no. 9, 2011.

[5] M. Hariharan, K. Polat, and R. Sindhu, "A new hybrid intelligent system for accurate detection of Parkinson's disease," Comput. Methods Programs Biomed., vol. 113, pp. 904-913, 2014. 
[6] W. Caesarendra, Farika T Putri, Mochammad Ariyanto and Joga D Setiawan, "Pattern Recognition Methods for Multi Stage Classification of Parkinson's Disease Utilizing Voice Features," IEEE International Conference of Advance Intelligent Mechatronic (AIM), 2015.

[7] M.A. Little, P.E. McSharry, E.J. Hunter, J. Spielman, and L.O. Ramig, "Suitability of dysphonia measurements for telemonitoring of Parkinson's disease," IEEE Trans. Biomed. Eng., vol. 56, no. 4, pp. 1015-1022, April 2009.

[8] M. Ariyanto, W. Caesarendra, K.A. Mustaqim, M. Irfan, J.A. Pakpahan, and J.D. Setiawan, "Finger Movement Pattern Recognition Method Using Artificial Neural Network Based on Electromyography (EMG) Sensor," International Conference on Automation, Cognitive Science, Optics, Micro Electro-Mechanical System and Information Technology (ICACOMIT), 2015.

[9] D. Tkach, H. Huang, T. Kuiken, "A Study of stability of time-domain features for electromyographic pattern recognition," J. Neuroeng. Rehabil., vol. 7(21), 2010.

[10] M. Zardoshti-Kermani, B.C. Wheeler, K. Badie, R.M. Hashemi, "EMG feature evaluation for movement control of upper extren mity prostheses," IEEE Trans. Rehabil. Eng.., vol. 3, pp. 324-333, Desember 1995.

[11] M.A. Oskoei, H. Hu. "Support vector machine based classification scheme for myoelectric control applied to upper limb," IEEE Trans..Biomed. Eng., vol. 55(8), pp. 1956-1965, August 2008.

[12] A. Phinyomark, C. Limsakul, P. Phukpattaranont, "A novel feature extraction for robust EMG pattern recognition," J. Comput, vol. 1(1), pp.71-80, Desember 2009.

[13] O. Dehzangi, Y. Zou and R. Jafari, "Simultaneous classification of motor imagery and SSVEP EEG signals," Neural Engineering (NER), $20136^{\text {th }}$ International IEEE/EMBS Conference on, San Diego, CA, 2013, pp. 1303-1306. 